\title{
4. An Electrical Phenomenon of a Palladium Filament Occluding Hydrogen.
}

\author{
By Kozo Hirota and Juro Horiuti.
}

(Comm. by M. KataYAMA, M.I.A., Jan. 12, 1939.)

It has long been known that the electrical resistance of a palladium wire was increased by occluded hydrogen. Coehn, Specht and Jürgens ${ }^{1)}$ have found that by applying the potential the occluded hydrogen could be shifted toward the negative terminal of the wire. These authors have suggested in this connection that occluded hydrogen was at least partly dissociated into constituent particles, i. e. protons and electrons. Franck ${ }^{2}$ has discussed the energetics of the dissociation process suggesting that these protons are shielded by electron clouds like Debye's ion in the solution, leaving more or less effective charges.

The points of view seems to be in accord with our new observations, to be reported in this paper, (1) on the dependence of resistance upon the quantity of occluded hydrogen and (2) on the after effect of the current through the filament, both being carried out with very thin filament and with an extremely small quantity of occluded hydrogen.

A palladium filament of $8 \mu$ diameter and of $1.0 \mathrm{~cm}$ length was cramped at both ends by copper wires and sealed in a glass cell of $8 \mathrm{cc}$ capacity, the latter being kept in a thermostat at $100^{\circ} \mathrm{C}$, connected to a vacuum line and electrically inserted in an arm of a Wheatstone bridge as shown in Fig. 1. Pd is the palladium cell, G the galvanometer, $K$ the key, $A B$ the slide resistance and $R_{1}$ and $R_{2}$ inductance free resistances.

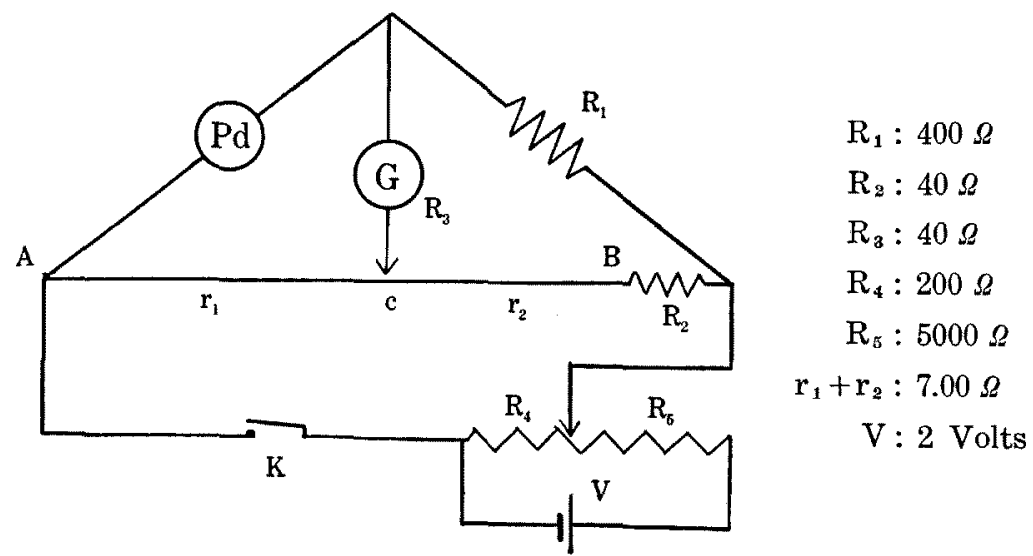

Fig. 1.

With the filament, observations (1) and (2) were made as follows: (1) The bridge was balanced with a momentary current caused by tapping the key. The apparent resistance of a freshly prepared

1) Coehn and Specht; Z. f. Phys., 62 (1930) 1 ; Coehn and Jürgens; ibid., 71, (1931) 179.

2) Franck ; Gött. Nachr. (1933) 293. 
cell, found initially $13.18 \Omega$, decreased gradually on continuous evacuation for 864 hours attaining a minimum at $11.35 \Omega$ and then increased again reaching finally a practically constant value $25.77 \Omega$. Next, by admitting hydrogen gas stepwise into the cell each time ca. $10^{-3} \mathrm{~mm} \mathrm{Hg}$, it was found that the former trend was reversed.

(2) The bridge was now balanced at first with a steady current of $2 \times 10^{-4}$ Ampere through the cell and then left for several tens of minutes with the circuit open. On again closing the circuit the galvanometer deflected in the sense apparently showing a decreased resistance of palladium below that of the balance. The deflection decreased immediately, the balance of the bridge being restored in a few minutes as shown in Fig. 2. On opening the circuit now the galvanometer deflected in the reversed sense but, within the limit of experimental error, by an equal amount to that before, the deflection again reducing to zero in a few minutes.

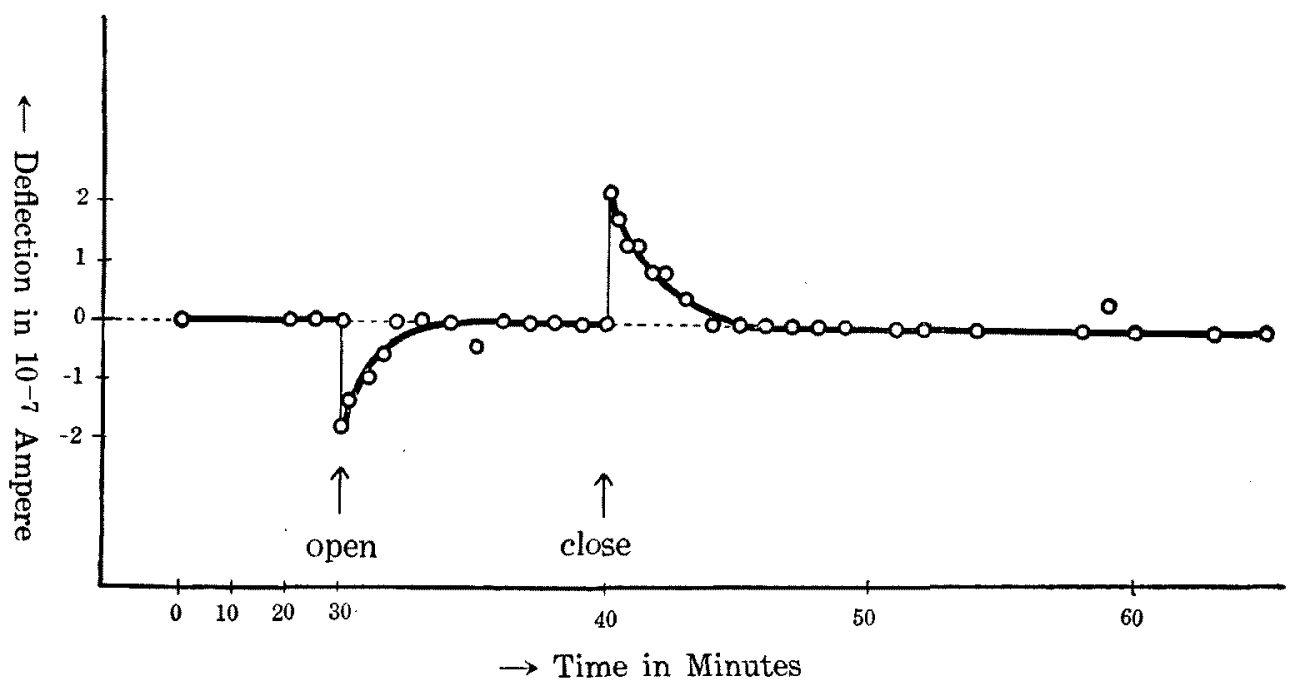

Fig. 2.

Since no other electromotive force was implied in any circuit through the galvanometer after opening the circuit at the key, we have to conclude that the filament, through which the current was passed, was responsible for the deflection.

These observations could adequately be accounted for as follows by postulating mobile protons in the metal, which retained, if shielded by electron clouds, more or less effective charges. Such protons will be referred to as "metal protons" in what follows.

On applying the potential to the palladium filament, metal protons migrate toward the end of the lower potential, where they are blocked by the practically impermeable copper wire. The migration ceases when the "sedimentation equilibrium" is set up, the applied potential being partly compensated by the reversed electromotive force due to the inhomogeneous distribution of the effective charges of the metal protons.

At the earlier stage of migration the protons may contribute to 
the conduction by attracting and accumulating electrons at the end of the lower potential ; this effect of apparently diminishing the resistance is more and more overcome towards the later stage by that of the gradually increasing reversed electromotive force, being survived by the latter at the "sedimentation equilibrium." The time required for setting up the equilibrium decreases with the concentration of the metal protons; for the larger the concentration the smaller shift of individual effective charges suffices to cancel the effect of the applied potential causing the migration.

By tapping the key for a finite time as in observation (1) the palladium wire would reveal features of its earlier stage of migration when the occluded hydrogen is dilute and those of the later stage when concentrated. This explains the results of observation (1) as well as those of earlier experiments in which the resistance was considerably increased by heavily occluded hydrogen of electrolysis.

Applying next a steady current through the filament the apparent resistance at every stage of migration will be continuously observed arriving finally at the balance as in the case of observation (2). Then by opening the key shifted protons will diffuse back to restore their homogeneous distribution. Some of the electrons may flow through the filament accompanying the protons while others through other parts of the net-work round, their proportion depending on the resistance involved. The latter part of the current will however flow mainly through the galvanometer in our case because of the arrangement of resistances, thus giving rise to the deflection in the opposite direction. The current should be equal in magnitude and opposite in sign to that at the moment of closing the circuit as observed, for, since the bridge was balanced with a steady current or at the "sedimentation equilibrium" of the metal protons, the situation at the moment of closing is equivalent to what would happen by inserting into the balanced circuit an electromotive force of equal amount and opposite sign to the reversed electromotive force at the balanced state, the latter prevailing alone on opening the circuit.

We are indebted to Dr. T. Sasahara of Kawanisi Factory in Kobe, who has kindly prepared for us the palladium cell used in the present research.

Our best thanks are due to the Gakuzyutu Sinkôkai and the Hattori-Hôkôkai for financial grants. 